\title{
Design of an efficient single photon source from a metallic nanorod dimer: a quasinormal mode finite-difference time-domain approach
}

\author{
Rong-Chun Ge* and S. Hughes \\ Department of Physics, Engineering Physics and Astronomy, Queen's University, Kingston, Ontario, Canada K' 3 L6 \\ ${ }^{*}$ Corresponding author: rchge@physics.queensu.ca \\ Compiled August 28, 2018
}

\begin{abstract}
We describe how the finite-difference time-domain (FDTD) technique can be used to compute the quasinormal mode (QNM) for metallic nano-resonators, which is important for describing and understanding light-matter interactions in nanoplasmonics. We use the QNM to model the enhanced spontaneous emission rate for dipole emitters near a gold nanorod dimer structure using a newly developed QNM expansion technique. Significant enhanced photon emission factors of around 1500 are obtained with large output $\beta$-factors of about $60 \%$. (C) 2018 Optical Society of America

OCIS codes: 240.6680, 160.4236, 270.5580.
\end{abstract}

Resonant cavity structures have the ability to trap light in very small spatial volumes which has a wide range of applications in nanophotonics [1]. Various miniaturized cavity structures have been developed over the years to manipulate light at the subwavelength scale, and extreme nanoscale confinement is now possible with metallic nano-resonators (MNRs). In a frequency regime near a localized surface plasmon (LSP) [2], the local density of optical states (LDOS) can be increased dramatically. Consequently, the spontaneous emission (SE) rate of a dipole emitter can be enhanced via the Purcell effect. The resonant enhancement from MNRs has applications in chemical sensing [3], high resolution imaging [4,5], optical antennas [6] and single photon emission [7].

While the optical properties of MNRs are being actively pursued, numerically modelling of the basic cavity physics is extremely demanding, and analytical solutions of the modes only exists for very simple structures such as spheres. For resonant cavity structures, the natural modes of the system are called quasinormal modes (QNMs) [8,9], defined as the frequency domain solutions to the wave equation with open boundary conditions (the Silver-Müller radiation condition). Kristensen et al. [10] first used the QNMs to introduce a rigorous definition of the "generalized effective mode volume" and Purcell factor [11], and applied these results to photonic cavity structures. For MNRs, the QNMs also form the natural starting point for developing analytical theories of lightmatter interactions in nanoplasmonics [12-14].

One of the most common numerical techniques for obtaining the cavity mode for dielectric cavities is the finite-difference time-domain (FDTD) technique [15]. The FDTD technique allows one to simulate open boundary conditions with "perfectly matched layers" (PMLs) located at the leaky mode region outside the cavity. For dielectric cavities, this open-boundary FDTD approach has been shown to yield excellent agreement with direct integral equation methods [10]. Other timedomain techniques such as the discontinuous Galerkin time-domain approach can also use PMLs [16]. For metals, a major problem occurs when using the usual mode calculation approach with a dipole excitation source, since the extracted mode depends sensitively on the dipole position [17], and is therefore incorrect. Thus it has been common practice to excite the MNR with a plane wave source and obtain the scattered field. However, this scattered field is not the same field as the QNM and it cannot be properly normalized for use in quantum optics, e.g., for obtaining the Purcell factor and effective mode volume [12] — two well known quantities that help describe the underlying physics of cavity light-matter interactions. While some frequency-domain techniques exist for computing the QNMs of MNRs $[18,19]$, it is highly desirable to be able to compute the QNMs using the commonly employed and general FDTD technique.

The FDTD method is already widely used by the plasmonics community, and its accuracy for obtaining the enhanced field has been verified against other numerical techniques such as the multipole expansion technique [20]. In addition, the LDOS can be calculated by employing a dipole excitation source [21-23], which can also model local field effects, e.g., associated with finite-size photon emitters inside a MNR [21]. While direct dipole calculations are feasible, they are very time consuming and require a new simulation (which may require many hours of computational time) for each spatial position of the dipole emitter; thus a QNM picture would be much more efficient, since it allows one to simulate dipole responses both as a function of space and frequency as soon as the QNM is obtained and properly normalized.

In this Letter we first describe how the FDTD technique [24] can be efficiently employed to obtain QNMs of a MNR by filtering the scattered field with a temporal window function. We compute the spatial dependence of the QNM and the effective mode volume, and show excellent agreement with full-dipole calculations for the enhanced SE factor of a dipole emitter. We then show how a gold nanorod dimer can act as an efficient single 
photon source with large Purcell factors (1500) and impressive output $\beta$-factors (around 60\%). In contrast to spherical dimer structures [25], we find that the nanorod dimer acts to increase the $\beta$-factor for good photon emitters (in comparison to a single resonator), and has the additional advantage of yielding resonant frequencies in the visible spectrum.

The QNM $\tilde{\mathbf{f}}_{\mu}$ has a complex eigenfrequency, $\tilde{\omega}_{\mu}=\omega_{\mu}-$ $\mathrm{i} \gamma_{\mu}$, with a quality factor $Q=\omega_{\mu} / 2 \gamma_{\mu}$. The QNM is normalized through $[8,9]$

$$
\begin{aligned}
\left\langle\left\langle\tilde{\mathbf{f}}_{\mu} \mid \tilde{\mathbf{f}}_{\nu}\right\rangle\right\rangle & =\lim _{V \rightarrow \infty} \int_{V}\left(\frac{1}{2 \omega} \frac{\partial\left(\epsilon(\mathbf{r}, \omega) \omega^{2}\right)}{\partial \omega}\right)_{\omega=\tilde{\omega}_{\mu}} \tilde{f}_{\mu}(\mathbf{r}) \cdot \tilde{\mathbf{f}}_{\nu}(\mathbf{r}) d \mathbf{r} \\
& +\frac{i c}{2 \tilde{\omega}_{\mu}} \int_{\partial V} \sqrt{\epsilon(\mathbf{r}) \tilde{\mathbf{f}}_{\mu}(\mathbf{r}) \cdot \tilde{\mathbf{f}}_{\nu}(\mathbf{r}) d \mathbf{r}=\delta_{\mu \nu}}
\end{aligned}
$$

Since the eigenfrequencies are complex, the QNMs diverge in space and each part of Eq. (1) diverges, but the total sum converges quickly in space $[12,14]$. This convergence occurs approximately when the outgoing field becomes purely oscillatory, rather than evanescent. For spatial points near the resonator, the transverse part of the photon Green function can be expanded as $[9,14] \mathbf{G}^{\mathrm{T}}\left(\mathbf{r}_{1}, \mathbf{r}_{2} ; \omega\right)=\sum_{\mu} \frac{\omega^{2}}{2 \tilde{\omega}_{\mu}\left(\tilde{\omega}_{\mu}-\omega\right)} \tilde{\mathbf{f}}_{\mu}\left(\mathbf{r}_{1}\right) \tilde{\mathbf{f}}_{\mu}\left(\mathbf{r}_{2}\right)$. One can then derive the effective mode volume $V_{\text {eff }}$ and enhanced SE factor $F_{a}\left(\mathbf{r}_{a}, \omega\right)$, where $\mathbf{r}_{a}$ is the spatial position of a dipole emitter. For dipole positions near the resonator, these quantities are defined through $[10,14]$

$$
\frac{1}{V_{\mathrm{eff}}}=\operatorname{Re}\left\{\frac{1}{v_{\mathrm{Q}}}\right\}, \quad v_{\mathrm{Q}}=\frac{\left\langle\left\langle\tilde{\mathbf{f}}_{\mathrm{c}} \mid \tilde{\mathbf{f}}_{\mathrm{c}}\right\rangle\right\rangle}{\varepsilon_{\mathrm{B}} \tilde{\mathbf{f}}_{\mathrm{c}}^{2}\left(\mathbf{r}_{0}\right)},
$$

and

$$
F_{a}\left(\mathbf{r}_{a}, \omega\right)=F_{\mathrm{P}} \eta\left(\mathbf{r}_{a}, \mathbf{n}_{a} ; \omega\right)+1,
$$

where $F_{\mathrm{P}} \eta\left(\mathbf{r}_{\mathrm{a}}\right)=\frac{3 Q}{4 \pi^{2}} \frac{\lambda_{\mathrm{c}}^{3}}{n_{\mathrm{B}}^{3}} \frac{\omega_{\mathrm{c}}^{2} \gamma_{\mathrm{c}}}{\omega} \operatorname{Im}\left[\frac{\mathbf{n}_{a} \varepsilon_{\mathrm{B}} \tilde{\mathbf{f}}_{\mathrm{c}}\left(\mathbf{r}_{a}\right) \tilde{\mathbf{f}}_{\mathrm{c}}\left(\mathbf{r}_{a}\right) \mathbf{n}_{a}}{\tilde{\omega}_{\mathrm{c}}\left(\tilde{\omega}_{\mathrm{c}}-\omega\right)}\right]$ is the Purcell factor, $F_{\mathrm{P}}$, multiplied by a factor $\eta$ to account for any deviations at $\mathbf{r}_{a}$ from the field maximum $\mathbf{r}_{0}$, cavity polarization, and cavity resonant frequency [14]; here $\mathbf{n}_{a}$ is the unit vector in the direction of the dipole emitter, $n_{\mathrm{B}}$ is the background-medium refractive index in which the MNR is embedded and $\lambda_{c}$ is the corresponding wavelength. Equation (3) assumes a single QNM expansion, which is valid for the MNR light-matter interaction regimes that we study below.

The FDTD technique can also obtain the enhanced SE factor directly, but only at the dipole position, which can be used to check the numerical accuracy of the QNM equations. Specifically, one can define [26]

$$
F_{a}^{\mathrm{FDTD}}\left(\mathbf{r}_{a}, \omega\right)=\frac{\operatorname{Im}\left\{\mathbf{n}_{a} \cdot \mathbf{G}^{\mathrm{FDTD}}\left(\mathbf{r}_{a}, \mathbf{r}_{a} ; \omega\right) \cdot \mathbf{n}_{a}\right\}}{\operatorname{Im}\left\{\mathbf{n}_{a} \cdot \mathbf{G}_{\mathrm{B}}\left(\mathbf{r}_{a}, \mathbf{r}_{a} ; \omega\right) \cdot \mathbf{n}_{a}\right\}},
$$

where $\mathbf{G}_{\mathrm{B}}$ is the known homogeneous medium Green function [26], and $\mathbf{G}^{\mathrm{FDTD}}\left(\mathbf{r}_{a}, \mathbf{r}_{a} ; \omega\right)$ is obtained from FDTD at the dipole source position [22]. Note that our full-dipole FDTD calculations have been shown elsewhere to yield good agreement with analytical results for metal spheres [27] (within 5\% agreement over broad- band frequencies and various spatial positions).

Next we discuss how FDTD is usually used to obtain resonator cavity modes and highlight the main problem for metallic structures. As a direct space-time simulation method, FDTD can obtain the spectral response of system from a Fourier transform of its time response. Thus for any incident field with a finite bandwidth, the computed scattered field, $\mathbf{E}^{\mathrm{S}}(\omega)$, will contain the resonant responses of the system, e.g., spectral peaks corresponding to the QMNs. Defining the total electric field as $\mathbf{E}^{\text {tot }}(\omega)$ (including the incident source), then the scattered field is simply $\mathbf{E}^{\mathrm{S}}(\omega)=\mathbf{E}^{\text {tot }}(\omega)-\mathbf{E}^{\mathrm{h}}(\omega)$, where $\mathbf{E}^{\mathrm{h}}(\omega)$ is the homogeneous solution without the MNR (i.e., no scattering). For a typical dielectric cavity with a high $Q$ resonance, almost any incident field or dipole source field can be used to obtain the mode of interested if one applies a temporal window to subtract off the excitation source. A common method of choice is to use a dipole excitation source and begin the Fourier transform of the scattered field after the excitation pulse has gone, which has been shown to yield good agreement with other mode solving techniques [10]. However, for a metal, the dipole source does not efficiently excite the QNM because of large losses, and extra care is needed in applying a temporal window function to compute the QNM of interest. Below we describe how the FDTD technique can obtain the true QNM, if the QNM is spectrally well isolated.

To cover a broad spectral range, we use a plane-wave excitation source with a 6 fs time duration. To efficiently excite the QNM, we choose the polarization of the source field to be parallel to the expected polarization of the QNM (e.g., along the rod axis for a nanorod). After obtaining the scattered field, we compute the QNM from

$$
\tilde{\mathbf{f}}_{\mathrm{c}}\left(\mathbf{r} ; \omega_{\mu}\right)=\int_{0}^{t_{\mathrm{end}}} \mathbf{E}^{\mathrm{S}}(\mathbf{r}, t) e^{i \omega_{\mathrm{c}} t} e^{-\frac{\left(t-t_{\text {off }}\right)^{2}}{2\left(\tau_{\mathrm{win}}\right)^{2}}} d t,
$$

where $\omega_{\mathrm{c}}$ is the real part of the eigenfrequency $\tilde{\omega}_{\mathrm{c}}, 2 \sqrt{\ln 2} \tau_{\text {win }}$ gives the FWHM (full-width at halfmaximum) of the time window, and $t_{\text {off }}$ is the time offset from the center of the source pulse to the center of the time windowing function. One criterion for the selection of time window parameters is to set $\omega_{\mathrm{c}} \tau_{\text {win }}^{2} / 2 t_{\mathrm{off}} \rightarrow Q$, while keeping $\tau_{\text {win }}$ as large as possible. To understand why this works, consider the ideal case for which the integral in Eq. (5) is carried out from $-\infty$ to $\infty$, and assume the scattered field is given by $\mathbf{E}^{\mathrm{S}}(t)=\sum_{i} \mathbf{E}_{i} e^{\left(-i \omega_{i}-\gamma_{i}\right) t}$ $\left(\left(\omega_{i}, \gamma_{i}\right) \neq\left(\omega_{j}, \gamma_{j}\right)\right.$ if $\left.i \neq j\right)$; after performing the integration, the amplitude of the $i$ th component is proportional to $\left|\mathbf{E}_{i} e^{\frac{\left(\mathrm{i} \Delta \omega_{i}-\Delta \gamma_{i}\right)^{2} \tau_{\text {win }}^{2}}{2}}\right| e^{-\left(\frac{t_{\text {off }}}{\tau_{\text {win }}}\right)^{2}}$ with $\Delta \omega_{i}=\omega_{i}-\omega_{\text {c }}$ and $\Delta \gamma_{i}=\gamma_{i}-t_{\text {off }} / \tau_{\text {win }}^{2}$. If the resonances of the system are well separated, so that $\left|\Delta \omega_{i}\right| \tau_{\text {win }} \gg 1$, and $\left|\Delta \omega_{i}\right| \gg\left|\Delta \gamma_{i}\right|$ for $i \neq \mathrm{c}$, then the QNM resonance, $\tilde{\omega}_{\mathrm{c}}=\omega_{\mathrm{c}}-\mathrm{i} \gamma_{\mathrm{c}}$, will be the only surviving term after applying the windowing function. This is achieved by having $t_{\text {off }} / \tau_{\text {win }}^{2}=\gamma_{\mu}$ for the QNM of interest; however, due to the factor $e^{-\frac{t_{\text {off }}}{\tau_{\text {win }}}}$, $t_{\text {off }}$ should not be too large, otherwise the spectrum will be too weak and influenced by numerical errors. We have 


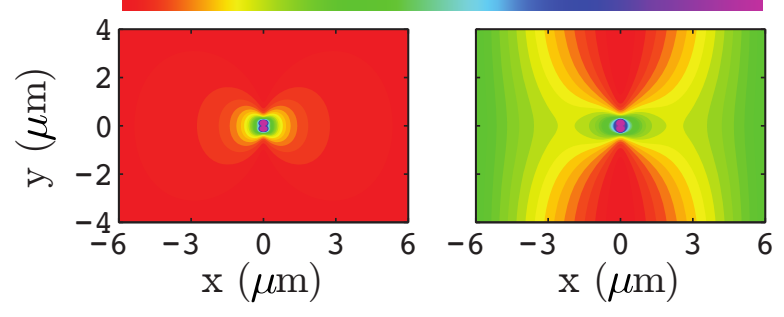

Fig. 1. Spatial profile of the dipole mode of a gold nanorod dimer, showing both the scattered field and QNM. Left: $\left|\mathbf{E}^{\mathrm{S}}\left(x, y, 0 ; \omega_{\mathrm{c}}\right)\right|$, and right: $\left|\tilde{\mathbf{f}}\left(x, y, 0 ; \omega_{\mathrm{c}}\right)\right|$ at $\omega_{\mathrm{c}}=291.06 \mathrm{THz}$. The excitation source is a $y$-polarized plane wave incident in the $x$ direction.

found that a good choice is to simply choose $t_{\text {off }}=4 \pi / \gamma_{\mathrm{c}}$, i.e., two times the lifespan of the QNM.

Next we apply the above technique to investigate a gold dimer structure made up of two identical nanorods. We choose a rod radius $\mathrm{r}_{a}=15 \mathrm{~nm}$ with an axis length $l=100 \mathrm{~nm}$ (along $y$ ). We use the Drude model, $\varepsilon(\omega)=1-\frac{\omega_{\mathrm{p}}^{2}}{\omega(\omega+\mathrm{i} \gamma)}$, with parameters similar to gold, with $\omega_{\mathrm{p}}=1.26 \times 10^{16} \mathrm{rad} / \mathrm{s}$ (plasmon frequency), and $\gamma=1.41 \times 10^{14} \mathrm{rad} / \mathrm{s}$ (collision rate). In order to get a larger enhancement of the SE for a quantum dipole, we consider two nanorods that are parallel with each other. The separation gap is set to $20 \mathrm{~nm}$, which helps minimizes nonradiative decay and also allows sufficient space to embed a quantum emitter such as a quantum dot (see dipole arrow in Fig. 2). From FDTD analysis, we find the dipole mode is around $\tilde{\omega}_{\mathrm{c}} / 2 \pi=291.06-\mathrm{i} 20.28 \mathrm{THz}$ $\left(\omega_{\mathrm{c}} \approx 1.2 \mathrm{eV}\right)$, which is redshifted (by about $34 \mathrm{THz}$ ) with respect to a single nanorod [14] due to the bonding effect; the corresponding quality factor is $Q \approx 7.2$, which is smaller than that for a single nanorod $\left(Q_{\text {single }} \approx 10\right)$.

To obtain the scattered field and the QNM, a $y$ polarized plane wave with frequency $\omega / 2 \pi=291.06 \mathrm{THz}$ is employed, incident in the $x$ direction. The simulation domain size is $12 \times 8 \times 6 \mu \mathrm{m}^{3}$ (Fig. 1) and $2.4 \times 2.4 \times 2.4 \mu^{3}$ (Fig. 2), and we use a conformal meshing scheme with a maximum step size $40 \mathrm{~nm}$ in all directions; a smaller refined mesh of $1 \mathrm{~nm}$ is used around the metal dimer; 100 layers of PML have been used with symmetric (antisymmetric) boundary condition in $z(y)$ direction for both simulation and the time step is 1.8875 as. We use a time window with parameters $t_{\text {off }}=100 \mathrm{fs}$ and $\tau_{\text {win }}=23.3 / \sqrt{\ln 2}$ fs. The left and right panels of Fig. 1 show the scattered field and QNM, respectively. As anticipated, the QNM is seen to have an increasing field value for positions further away from the resonator, which is caused by the outgoing boundary conditions and the complex eigenfrequency. A close-up view of the QNM near the resonator is shown in the left panel of Fig. 2, and the correspondent mode volume is calculated to be $V_{\text {eff }} \approx 1.9 \times 10^{-4}\left(\lambda_{c} / n_{B}\right)^{3}$ (which is about double that of the single nanorod). The oscillating charge distribution is of opposite sign at both ends of each nanorod; also, the oscillating charge at the bottom of the upper nanorod

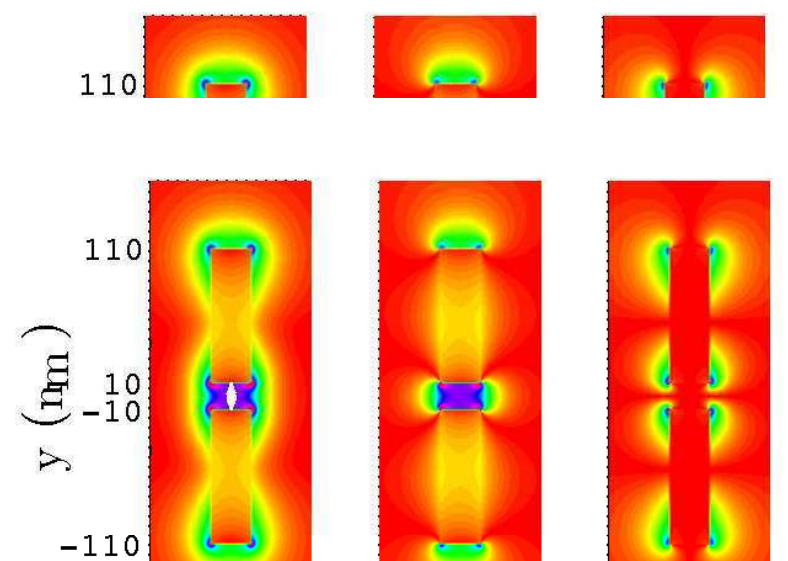

Fig. 2. Close up view of the QNM profile of the gold dimer. Left: $\left|\tilde{\mathbf{f}}\left(x, y, 0 ; \omega_{\mathrm{c}}\right)\right|$, middle: $\left|\tilde{\mathbf{f}}_{y}\left(x, y, 0 ; \omega_{\mathrm{c}}\right)\right|$, and right: $\left|\tilde{\mathbf{f}}_{x}\left(x, y, 0 ; \omega_{\mathrm{c}}\right)\right|$ at $\omega_{\mathrm{c}}=291.06 \mathrm{THz}$. A $y$-polarized dipole at the center of the gap is shown by the white dot and double arrow in the left panel.

has a different sign from the charge at the top of the lower nanorod, which makes the localized surface plasmons of the individual nanorods couple effectively. Consequently, the field inside the cavity (gap) is significantly enhanced and $y$-polarized. The middle and right panels in Fig. 2 show the $y$ and $x$ components of the QNM at $z=0$. The node of the $y$-component sits around both ends of the nanorods, slightly inside this metal. Similar nodal lines appear for an electric dipole composed of charge $\mp q$.

We remark that for these calculations it is important to choose a $y$-polarized plane wave, which efficiently excites the QNM of interest. If we use an $x$-polarized plane wave, with the parameters same as above, then a rather strange pseudo-mode is obtained. The failure of using $x$-polarized plane wave to obtain the QNM can be explained by the fact that the QNM can not be efficiently excited since the dipole moment lies along the $y$ direction. So some care is needed in choosing the correct excitation source, though in practice this is easy to do when exciting the QNM of interest. We have also checked that our mode calculation approach successfully reproduces the mode profile and QNMs for dielectric cavity structures, e.g., in Ref. 10 and also for other MNRs.

Single photon enhancement factors. As a possible application of using MNRs for single photon emitters, a large enhancement of the SE factor is desired. This can be achieved by having a quantum dipole at the gap center of the dimer. In Fig. 3, the enhanced SE factor, $F_{y}$, of a $y$ polarized dipole emitter at the center of the gap is shown by the blue (dashed) curve using a full-dipole numerical calculation with no approximations, i.e., $F_{y}^{\text {FDTD }}[22,23]$. Next we use the QNM to obtain the enhanced SE factor [14]. The result is shown by the orange (solid) curve in Fig. 3; it agrees extremely well with the full-dipole FDTD calculations. Indeed, the agreement also confirms that the entire response is dominated by a single QNM 


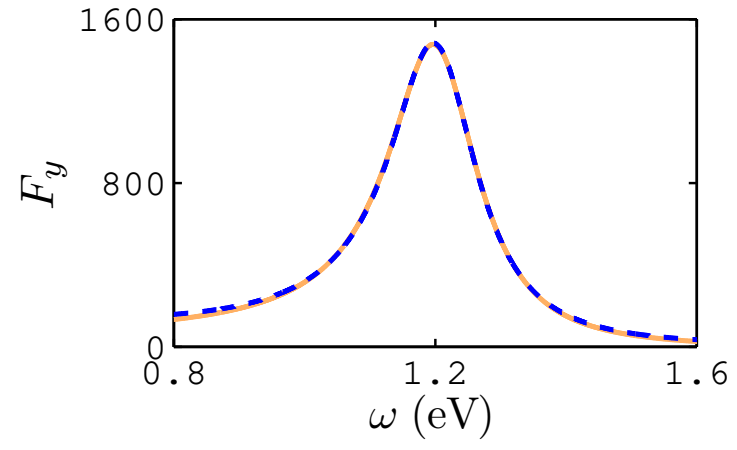

Fig. 3. Enhanced SE factor for the gold nanorod dimer structure, with the blue (dashed) curve given by direct FDTD calculation (Eq. (4)) and the orange (solid) curve given by the mode expansion technique (Eq. (3)). The emission dipole is $y$-polarized at the center of the gap of the dimer (see left panel of Fig. 2.)

which is highly desired for single photon source applications. From our results, we obtain impressive peak emission factors of around 1500. Note that although the $Q / V$ factor of the dimer structure is about three times smaller than the single nanorod, the emission factor of the dimer is around three times larger than for a single nanorod at an equivalent dipole position. Thus the $Q / V$ is clearly not the main metric to describe the enhanced emission factors for dipole away from the field antinode; note the reason for not choosing the field antinode, is that the emission would be severely quenched from Ohmic heating and a single mode Purcell factor ceases to have any meaning [14]. In this regard, the other important figure-of-merit for emitting single photons is the output-coupling $\beta$ factor (probability of photon emission via radiative decay in the far field) of the dipole emitter, which is computed here to be $\beta \approx 58 \%$; this again is better than the single gold nanorod, by about $10 \%$; with respect to single nanorod, the electric field of the dimer is repelled outside the lossy nanorods (especially around the hot spots) due to opposite charge distribution between them. This leads an overall nonradiative decay reduction $\left(\propto \int \operatorname{Im}[\epsilon(\mathbf{r}, \omega)]\left|\mathbf{E}^{\text {tot }}(\mathbf{r}, \omega)\right|^{2} d V\right)$, even though the volume of the integral is doubled.

In summary, we have described how the commonly employed FDTD method can be used to efficiently obtain the QNMs for metallic resonators by applying a filtering function to the scattered field. These calculations are verified by comparing the resulting $\mathrm{SE}$ emission factor with full-dipole FDTD numerical calculations, where we find excellent agreement. Using this technique, we have proposed a metal nanorod dimer structure that acts an efficient single photon source, yielding a large Purcell factor of 1500 and an output $\beta$ factor of around $60 \%$.

This work was supported by the Natural Sciences and Engineering Research Council of Canada. We thank Jeff Young and Philip Kristensen for useful discussions.

\section{References}

1. R. K. Chang and A. J. Campillo, Optical Proesses in Microavities, (World Scientific,1996).

2. S. A. Maier, Plasmonics: Fundamentals and Appliations (Springer, 2007).

3. K. A. Willets and R. P. Van Duyne, Ann. Rev. Phys. Chem. 58, 267 (2007).

4. K. Kneipp, Y. Wang, H. Kneipp, L. T. Perelman, I. Itzkan, R. R. Dasari, and M. S. Feld, Phys. Rev. Lett. 78, 1667 (1997).

5. R. Zhang, Y. Zhang, Z.-C. Dong, S. Jiang, C. Zhang, L. G. Chen, L. Zhang, Y. Liao, J. Aizpurua, Y. Luo, J.-L. Yang, and J.-G. Hou, Nature 498, 82 (2013).

6. P. Bharadwaj, B. Deutsch, and L. Novotny, Adv. Opt. Photon. 1, 438 (2009).

7. C. Belacel, B. Habert, F. Bigourdan, F. Marquier, J.-P. Hugonin, S. M. de Vasconcellos, X. Lafosse, L. Coolen, C. Schwob, C. Javaux, B. Dubertret, J.-J. Greffet, P. Senellart, and A. Maitre, Nano Lett. 13, 1516 (2013).

8. P. T. Leung, S. Y. Liu, and K. Young, Phys. Rev. A 49, 3982 (1994).

9. K. M. Lee, P. T. Leung, and K. M. Pang, J. Opt. Soc. Am. B 16, 1409 (1999).

10. P. T. Kristensen, C. Van Vlack, and S. Hughes, Opt. Lett. 37, 1649 (2012).

11. E. M. Purcell, Phys. Rev. 69, 674 (1946).

12. P. T. Kristensen and S. Hughes, ACS Photonics 1, 2-10 (2014).

13. C. Sauvan, J. P. Hugonin, I. S. Maksymov, and P. Lalanne, Phys. Rev. Lett. 110, 237401 (2013).

14. R.-C. Ge, P. T. Kristensen, Jeff. F. Young, S. Hughes, e-print: arXiv:1312.2939 [quant-ph]

15. A. Taflove and S. C. Hagness, Computational Electrodynamics, Artech House, 2005.

16. e.g., see K. Stannigel, M. König, J Niegemann, and K. Busch, Optics Express 17, 14934 (2009).

17. Kevin C. Y. Huang, Young Chul Jun, Min-Kyo Seo, and Mark L. Brongersma, Opt. Express 19, 19084 (2011).

18. Q. Bai, M. Perrin, C. Sauvan, J-P Hugonin, and P. Lalanne, Opt. Express 22 , 27371 (2013).

19. J. R. de Lasson, J. Mørk, and P. T. Kristensen, J. Opt. Soc. Am. B 30, 19967 (2013).

20. A. Dhawan, S. J. Norton, M. D. Gerhold, and T. VoDinh, Opt. Express 17, 9688 (2009).

21. C. Van Vlack and S. Hughes, Opt. Lett. 37, 2880 (2012).

22. P. Yao, V.S.C. Manga Rao, and S. Hughes, Laser and Photonics Reviews 4, 499 (2010).

23. V.S.C. Manga Rao and S. Hughes, Phys. Rev. Lett. 99, 193901 (2007).

24. We use FDTD Solutions: www.lumerical.com.

25. G. Sun and J. B. Khurgin, Appl. Phys. Lett. 98, 113116 (2011).

26. L. Novotny and B. Hecht, Principles of Nano Optics, Cambridge University Press, 2006.

27. C. Van Vlack, P. T. Kristensen, and S. Hughes, Phys. Rev. B 85, 075303 (2012). 